\title{
ADUBO DE LIBERAÇÃO LENTA NA PRODUÇÃO DE MUDAS DE MAMOEIRO ${ }^{1}$
}

\author{
LUIZ AUGUSTO LOPES SERRANO ${ }^{2}$, LAERCIO FRANCISCO CATTANEO ${ }^{2}$ \\ GERALDO ANTÔNIO FERREGUETTI ${ }^{3}$
}

RESUMO - O objetivo deste trabalho foi definir as doses adequadas de um adubo de liberação lenta para a produção de mudas dos principais genótipos de mamoeiro cultivados no Estado do Espírito Santo. O experimento foi realizado em estufa agrícola, na Empresa Caliman Agrícola S.A., em Linhares-ES, sob delineamento de blocos casualizados, em esquema fatorial $5 \times 6$. Foram avaliados cinco genótipos de mamoeiro 'Golden', 'Calimosa', 'Tainung 01', 'INCAPER 09' e 'INCAPER 39', cultivados em substrato comercial fertilizado com seis doses de um adubo de liberação lenta, fórmula NPK + $(\mathrm{Mg})$ 13-06-16 + $(1,4)$ : 0,$0 ; 2,5 ; 5,0 ; 7,5 ; 10,0$ e $12,5 \mathrm{~kg} \mathrm{~m}^{-3}$. Houve diferenças no crescimento e no estado nutricional das mudas de mamoeiro em relação ao genótipo e às doses do adubo de liberação lenta utilizados. As doses do adubo que proporcionaram os maiores valores para altura e massa seca total das mudas de mamoeiro foram: 11,2 e 11,3 $\mathrm{kg} \mathrm{m}^{-3}$ para o 'Golden'; 7,7 e 7,9 $\mathrm{kg} \mathrm{m}^{-3}$ para o 'Calimosa'; 11,6 e 10,9 $\mathrm{kg} \mathrm{m}^{-3}$ para o 'Tainung 01'; 10,5 e $10,6 \mathrm{~kg} \mathrm{~m}^{-3}$ para o 'INCAPER 09', e 11,0 e 9,6 $\mathrm{kg} \mathrm{m}^{-3}$ para o 'INCAPER 39', respectivamente. Nessas mesmas doses do adubo, as mudas apresentaram adequado nível nutricional.

Termos para indexação: Carica papaya L., nutrição mineral de plantas, matéria seca total.

\section{CONTROLLED-RELEASE FERTILIZER IN PAPAYA SEEDLINGS PRODUCTION}

\begin{abstract}
The objective of this work was to define the appropriate doses of a controlled-release fertilizer in the production of papaya seedlings, in the state of Espirito Santo, Brazil. The research was realized in a greenhouse in Caliman Agricola Company, Linhares- ES, Brazil. The experimental design was a randomized complete block in a factorial scheme 5x6. It was evaluated five papaya genotypes 'Golden', 'Calimosa', 'Tainung 01', 'INCAPER 09' and 'INCAPER 39', cultivated in commercial substrate (constituted of pinus bark and vermiculite) fertilized with six doses of controlled-release fertilizer formula NPK $+(\mathrm{Mg})$ 13-06$16+(1.4): 0.0 ; 2.5 ; 5.0 ; 7.5 ; 10.0$ and $12.5 \mathrm{~kg} \mathrm{~m}^{-3}$. Differences in growth and nutritional status of papaya seedlings were observed according to the genotype and the dose of the controlled-release fertilizer used. The highest values for height and total dry matter of papaya seedlings occurred with the doses: 11.2 and $11.3 \mathrm{~kg} \mathrm{~m}^{-3}$ for 'Golden'; 7.7 and $7.9 \mathrm{~kg} \mathrm{~m}^{-3}$ for 'Calimosa'; 11.6 and $10.9 \mathrm{~kg} \mathrm{~m}^{-3}$ for 'Tainung 01'; 10.5 and $10.6 \mathrm{~kg} \mathrm{~m}^{-3}$ for 'INCAPER 09' and 11.0 and $9.6 \mathrm{~kg} \mathrm{~m}^{-3}$ for 'INCAPER 39', respectively. In the same doses the papaya seedlings showed adequate levels of nutrients.
\end{abstract}

Index terms: Carica papaya L., nutritional mineral of plants, total dry matter.

\section{INTRODUÇÃO}

No Estado do Espírito Santo, o cultivo do mamoeiro (Carica papaya L.) é realizado numa área superior a 9.300 ha, tendo como destaques os municípios de Pinheiros e Linhares, maiores produtores estaduais de mamões dos grupos 'Formosa' e 'Solo', respectivamente (IBGE, 2008).

Um dos fatores que têm preocupado os produtores capixabas são as doenças de solo causadas por fungos que ocorrem no viveiro de mudas ou em mudas recém-transplantadas no campo, como o estiolamento, causado pelos fungos Pythium sp., Sclerotium sp., Fusarium sp. e Rhizoctonia sp., e a podridão-do-pé ou gomose, causada pelo fungo Phytophthora palmivora (VENTURA et al., 2006). Essas doenças ocasionam acréscimos nos gastos com mudas e com mão de obra devido à necessidade de replantio no campo.

A maioria dos produtores de mudas de mamoeiro do Estado do Espírito Santo utiliza como substrato misturas que contêm solo, geralmente não expurgado, aumentando a possibilidade da disseminação de patógenos de solo para toda a região

\footnotetext{
${ }^{1}$ (Trabalho 120-09). Recebido: 15-05-2009. Aceito para publicação: 27-05-2010.

${ }^{2}$ Eng. ${ }^{\circ}$ Agrônomo, D.Sc., Pesquisador do Instituto Capixaba de Pesquisa, Assistência Técnica e Extensão Rural (INCAPER), Rodovia BR 101, Km 151, Caixa Postal 62, CEP: 29915-140, Linhares-ES. E-mails: lalserrano@incaper.es.gov.br; lfcattaneo@hotmail.com ${ }^{3}$ Eng. ${ }^{\circ}$ Agrônomo, Diretor Agrícola da Caliman Agrícola S/A, BR 101, Km 111, Fazenda Santa Terezinha, Caixa Postal 52, 29900-970, Linhares-ES. E-mail: geraldo@caliman.com.br.
} 
produtora.

A utilização de substratos comerciais (compostos principalmente por materiais orgânicos e vermiculita) em substituição ao substrato convencional (composto por solo, areia e esterco) apresenta inúmeras vantagens, como melhor aeração e drenagem; adequados valores de $\mathrm{pH}$ e salinidade, otimizando a absorção de água e nutrientes pela raiz; baixa densidade, facilitando e aumentando o rendimento do transporte; homogeneidade do material; disponibilidade constante no mercado; melhor aproveitamento e rendimento da mão de obra, devido à praticidade no preparo e no enchimento dos recipientes, e, principalmente, ausência de pragas e propágulos de doenças e plantas daninhas (KÄMPF, 2004).

Entretanto, na produção de mudas em substratos comerciais, há necessidade de aplicações frequentes de nutrientes, devido, principalmente, à sua lixiviação. Assim, a utilização de adubos que apresentam liberação controlada dos nutrientes tornase uma das alternativas para aumentar a eficiência das adubações.

Nos adubos de liberação lenta, os nutrientes são encapsulados por resinas especiais e são liberados mais lentamente, propiciando uma disponibilidade contínua dos mesmos para as plantas. Ao absorver os nutrientes, as raízes causam uma depleção na concentração dos nutrientes, nas proximidades da zona radicular, induzindo um sistema de liberação de nutrientes por osmose (TOMASZEWSKA et al., 2002). Semelhantemente à utilização de substratos comerciais, a utilização de adubos de liberação lenta também permite ao produtor de mudas uma redução de gastos com mão de obra com a aplicação de fertilizantes, uma vez que, para determinadas espécies, é necessário apenas a mistura deste adubo ao substrato (PEREIRA et al., 2000; LANA et al., 2002; SERRANO et al., 2006).

No norte capixaba, os produtores de mudas de mamoeiro que utilizam substrato comercial realizam diferentes manejos de adubação, sendo que tanto as formulações quanto as quantidades dos fertilizantes utilizados são muito variáveis. Nesta região, parte destes produtores utiliza um determinado adubo de liberação lenta, fórmula NPK (Mg) 13-06-16 (1,4), na dose de $1,0 \mathrm{~kg} \mathrm{~m}^{-3}$, associado a pulverizações foliares com adubos de várias formulações em intervalos de três dias, perfazendo cerca de 10 pulverizações durante a fase de produção de mudas ( $\approx 30$ dias $)$. Outros produtores utilizam este mesmo adubo de liberação lenta em doses de até $15 \mathrm{~kg} \mathrm{~m}^{-3}$ misturados ao substrato, com e sem aplicação de adubo foliar. Em ambos os casos, as doses utilizadas são fixas para qualquer genótipo de mamoeiro e para qualquer tipo (formulação) de adubo de liberação lenta.

Atualmente, não se encontram na literatura padrões de referência para os teores de nutrientes na massa seca de mudas de mamoeiro, entretanto Malavolta et al. (1997) consideram adequados para plantas adultas de mamoeiro os teores no limbo foliar de 4,5 a 5,0 dag $\mathrm{kg}^{-1}$ de N; 0,5 a 0,7 dag $\mathrm{kg}^{-1}$ de P; 2,5 a 3,0 dag $\mathrm{kg}^{-1}$ de $\mathrm{K} ; 2,0$ a 2,2 dag $\mathrm{kg}^{-1}$ de $\mathrm{Ca}$; 1,0 dag $\mathrm{kg}^{-1}$ de $\mathrm{Mg} ; 0,4$ a 0,6 dag $\mathrm{kg}^{-1}$ de $\mathrm{S} ; 15 \mathrm{mg}$ $\mathrm{kg}^{-1}$ de B; $11 \mathrm{mg} \mathrm{kg}^{-1}$ de Cu; $291 \mathrm{mg} \mathrm{kg}^{-1}$ de Fe; 70 mg kg-1 de Mn, e $43 \mathrm{mg} \mathrm{kg}^{-1}$ de Zn. Em mamoeiro 'Improved Sunrise Solo 72/12', Almeida et al. (2002) constataram que a maior produção de frutos $(53 \mathrm{t}$ $\mathrm{ha}^{-1}$ ) ocorreu nas plantas que apresentaram no limbo foliar os teores de 4,37 dag $\mathrm{kg}^{-1}$ de N; $0,37 \mathrm{dag}_{\mathrm{kg}}{ }^{-1}$ de P; 2,09 dag $\mathrm{kg}^{-1}$ de $\mathrm{K} ; 1,62$ dag $\mathrm{kg}^{-1}$ de $\mathrm{Ca} ; 0,79$ dag kg ${ }^{-1}$ de Mg; 0,43 dag kg ${ }^{-1}$ de S; 44 mg kg $^{-1}$ de B; $5,2 \mathrm{mg} \mathrm{kg}^{-1} \mathrm{de} \mathrm{Cu} ; 134 \mathrm{mg} \mathrm{kg}^{-1} \mathrm{de} \mathrm{Fe} ; 121,3 \mathrm{mg} \mathrm{kg}^{-1}$ de $\mathrm{Mn}$, e $24,8 \mathrm{mg} \mathrm{kg}^{-1}$ de $\mathrm{Zn}$.

Alguns trabalhos mostram o potencial benéfico da utilização de substrato comercial (MENDONÇA et al., 2003; YAMANISHI et al., 2004) e do adubo de liberação lenta (MENDONÇA et al., 2004; YAMANISHI et al., 2004) na produção de mudas de mamoeiro. Assim, o objetivo deste trabalho foi avaliar quais as doses adequadas de um adubo de liberação lenta na produção de mudas dos principais genótipos de mamoeiro cultivados no Estado do Espírito Santo.

\section{MATERIAL E MÉTODOS}

$\mathrm{O}$ experimento foi realizado em estufa agrícola, na Empresa Caliman Agrícola S.A., em Linhares-ES, sob delineamento de blocos casualizados completos, em esquema fatorial $5 \times 6$. Os tratamentos foram dispostos em 3 canteiros (blocos), formados por bandejas com capacidade para 96 tubetes de $50 \mathrm{~cm}^{3}$. Em cada canteiro, havia uma parcela experimental de cada tratamento, composta por 40 plantas, das quais as 12 centrais foram avaliadas.

Foram avaliados cinco genótipos de mamoeiro: 'Golden' (linhagem pura do grupo ‘Solo', sendo o mais cultivado na região); 'Calimosa UENF/CALIMAN 01'e 'Tainung 01' (híbridos F1 do grupo 'Formosa', sendo que o primeiro é um material recém-lançado no mercado nacional e o segundo é um material importado, porém o mais cultivado na região); 'INCAPER 09' e 'INCAPER 39' (variedades elite do grupo 'Formosa', oriundas do Programa de Melhoramento Genético do INCAPER). 
As mudas foram produzidas em substrato comercial Plantmax $\mathrm{HT}^{\circledR}$, composto por casca de pínus e vermiculita. Através de análises químicas (SILVA, 1999), realizadas no Laboratório de Solos do INCAPER CRDR/CS, constatou-se que este substrato continha $47 \%$ de umidade; $47 \%$ de matéria orgânica; relação C:N de 34:1 e pH 5,1. Por meio de digestão úmida (sulfúrica e nitroperclórica), determinaram-se os teores de nutrientes deste substrato: $8,0 \mathrm{~g} \mathrm{~kg}^{-1} \mathrm{de}$ $\mathrm{N} ; 2,2 \mathrm{~g} \mathrm{~kg}^{-1}$ de P; $3,1 \mathrm{~g} \mathrm{~kg}^{-1}$ de $\mathrm{K} ; 8,7 \mathrm{~g} \mathrm{~kg}^{-1}$ de Ca; 2,8 $\mathrm{g} \mathrm{kg}^{-1}$ de Mg; 2,1 $\mathrm{g} \mathrm{kg}^{-1}$ de S; $18 \mathrm{mg} \mathrm{kg}^{-1}$ de Zn; $5.700 \mathrm{mg} \mathrm{kg}^{-1}$ de Fe; $182 \mathrm{mg} \mathrm{kg}^{-1}$ de Mn; $11 \mathrm{mg} \mathrm{kg}^{-1}$ de $\mathrm{Cu}$, e $15 \mathrm{mg} \mathrm{kg}^{-1}$ de $\mathrm{B}$.

Ao substrato, foram misturadas seis doses do adubo de liberação lenta Basacote mini $3 \mathrm{M}^{\circledR}$, fórmula NPK $(\mathrm{Mg})$ 13-06-16 $(1,4)$ com micronutrientes: 0,0; 2,$5 ; 5,0 ; 7,5 ; 10,0$ e $12,5 \mathrm{~kg} \mathrm{~m}^{-3}$. A escolha desse adubo deve-se ao motivo de ser o mais utilizado na região para a produção de mudas de diversas espécies (Carica papaya, Eucalyptus sp., Passiflora edulis f. flavicarpa, dentre outras). As garantias desse adubo de liberação lenta são $13 \%$ de N $(5 \%$ N-nítrico + 8\% de $\mathrm{N}$-amoniacal); $6 \%$ de $\mathrm{P}_{2} \mathrm{O}_{5} ; 16 \%$ de $\mathrm{K}_{2} \mathrm{O} ; 1,4 \%$ de $\mathrm{Mg} ; 10 \%$ de S, $0,26 \%$ de Fe; $0,02 \%$ de $\mathrm{B} ; 0,05 \%$ de $\mathrm{Cu} ; 0,06 \%$ de $\mathrm{Mn}$, e $0,015 \%$ de Mo (COMPO DO BRASIL, 2009).

A semeadura foi realizada em 09-05-2008, colocando-se 3 sementes por tubete. Aos 10 dias após a semeadura (DAS), já se tinha $100 \%$ de germinação para todas as parcelas, e aos $15 \mathrm{DAS}$, foi realizado o desbaste de mudas, deixando-se uma muda por tubete.

Durante todo o período experimental, foi realizada irrigação diária das mudas por meio do sistema de fita de microaspersão, também conhecido como sistema "Santeno". Quanto à aplicação de produtos via pulverização, houve uma única aplicação de fungicida à base de fosetyl-Al, aos 25 DAS.

Aos 30 DAS (época em que os produtores consideram as mudas aptas ao plantio no campo, pois a partir desse período as plantas começam a estiolar), foram avaliados a altura das mudas (do colo até a gema apical), o diâmetro do caule (a $0,5 \mathrm{~cm}$ do colo), o número de folhas e o comprimento da raiz. Nessa etapa da avaliação, foram avaliadas todas as mudas úteis do experimento (1.080 plantas), ou seja, 36 plantas de cada tratamento.

Posteriormente, as partes aéreas e os sistemas radiculares das mudas avaliadas foram separados e secos em estufa $\left(70^{\circ} \mathrm{C}\right.$ por 72 horas) para a determinação das massas secas da parte aérea, do sistema radicular e total, utilizando-se de uma balança de precisão. Em seguida, as partes aéreas secas das 12 plantas de cada parcela experimental foram reunidas para formar uma amostra por bloco de cada tratamento (três repetições), sendo assim encaminhadas para o Laboratório de Análises de Solos e Foliar do INCAPER CRDR/NE para a quantificação dos teores de $\mathrm{N}, \mathrm{P}, \mathrm{K}, \mathrm{Ca}, \mathrm{Mg}, \mathrm{S}, \mathrm{B}$, $\mathrm{Cu}, \mathrm{Fe}, \mathrm{Mn}$ e Zn (SILVA, 1999).

Os dados obtidos foram submetidos à análise de variância. As médias obtidas pelos genótipos foram comparadas pelo teste de Tukey, e o efeito das doses do adubo foi ajustado pela análise de regressão polinomial, ambos a $5 \%$.

\section{RESULTADOS E DISCUSSÃO}

Para todas as características de crescimento avaliadas, foram constatadas diferenças entre os genótipos e entre as doses do adubo de liberação lenta (Tabela 1). Já em relação à interação genótipo e doses do adubo, não houve diferença significativa apenas para o comprimento da raiz das mudas. As mudas dos mamoeiros 'Golden' e 'Calimosa' apresentaram os maiores valores de comprimento da raiz: 14,6 e 14,3 cm, respectivamente, enquanto as mudas do mamoeiro 'INCAPER 09' apresentaram o menor valor: $13,6 \mathrm{~cm}$. Quanto ao efeito das doses do adubo sobre o comprimento da raiz das mudas, foi observada uma resposta do tipo quadrática $\left(\mathrm{R}^{2}=\right.$ 0,88 ), sendo a dose de $7,4 \mathrm{~kg} \mathrm{~m}^{-3}$ a que proporcionou o valor máximo de $15,2 \mathrm{~cm}$.

Verificando as interações entre os genótipos de mamoeiro e as doses do adubo de liberação lenta, verificou-se que as mudas dos híbridos 'Tainung 01 ' e 'Calimosa' obtiveram os maiores valores de altura, diâmetro do caule e massas secas da parte aérea, do sistema radicular e total (Tabela 2). Yamanishi et al. (2004) também observaram que mudas do 'Tainung 01' apresentaram, para as mesmas condições de cultivo, médias de crescimento superiores às mudas de 'Sunrise Solo'. Neste experimento, esses dois genótipos são os únicos híbridos e, por pertencerem ao grupo 'Formosa', tendem a apresentar maior vigor, devido aos efeitos da heterose - fenômeno pelo qual os filhos apresentam melhor desempenho do que a média dos pais (DANTAS et al., 2002); e maior rusticidade (FRAIFE FILHO et al., 2001).

$\mathrm{O}$ crescimento médio obtido pelas mudas dos cinco genótipos de mamoeiros, aos 30 dias após a semeadura (DAS), foram superiores às médias observadas por Mendonça et al. (2003) aos 60 DAS, com a produção de mudas do mamoeiro 'Sunrise Solo' em tubetes $(50 \mathrm{~mL})$ preenchidos com Plantmax ${ }^{\circledR}$. As mudas do 'Tainung 01 ' que receberam doses do adubo acima de $5 \mathrm{~kg} \mathrm{~m}^{-3}$ apresentaram médias semelhantes às observadas por Yamanishi et al. (2004) aos 45 DAS, ao produzirem mudas deste 
genótipo em Plantmax ${ }^{\circledR}$ fertilizado com $5 \mathrm{~kg} \mathrm{~m}^{-3}$ de um adubo de liberação lenta, fórmula NPK 14-14-14.

Os resultados mostraram ainda que, para a obtenção das maiores médias de crescimento, as mudas do 'Calimosa' exigiram doses menores do adubo, enquanto as mudas do 'Golden' exigiram doses mais elevadas (Tabela 2). Desse modo, inferese que há diferença quanto à dose do adubo exigida para determinado genótipo, portanto o produtor de mudas não deve adotar uma dose fixa desse adubo para a produção de mudas de mamoeiro.

Os produtores de mudas de mamoeiro do Estado do Espírito Santo consideram a altura das mudas $(\approx 10 \mathrm{~cm})$ como principal indicativo do momento em que as mudas devem ser transplantadas para o campo. Entretanto, como o valor da altura das plantas pode ser influenciado por possíveis distorções provenientes do excesso de $\mathrm{N}$ e também pela competição por luminosidade (estiolamento), torna-se importante considerar, também, a massa seca total da planta (MARANA et al., 2008).

Assim, tanto para a altura quanto para a massa seca total das plantas, as doses do adubo de liberação lenta, fórmula NPK $(\mathrm{Mg})$ 13-06-16 $(1,4)$, que melhor se ajustaram para a produção de mudas de mamoeiro foram: 11,2 e $11,3 \mathrm{~kg} \mathrm{~m}^{-3}$ para o 'Golden'; 7,7 e 7,9 $\mathrm{kg} \mathrm{m}^{-3}$ para o 'Calimosa'; 11,6 e $10,9 \mathrm{~kg} \mathrm{~m}^{-3}$ para o 'Tainung 01'; 10,5 e 10,6 kg $\mathrm{m}^{-3}$ para o 'INCAPER 09', e 11,0 e 9,6 $\mathrm{kg} \mathrm{m}^{-3}$ para o 'INCAPER 39'.

Com relação aos teores de nutrientes na massa seca da parte aérea das mudas, foram constatadas diferenças entre os genótipos para todos os nutrientes avaliados (Tabela 3), corroborando Marinho et al. (2002), que afirmam que a capacidade de absorção de nutrientes e o aproveitamento final destes pelo mamoeiro diferem entre os genótipos.

As mudas do mamoeiro 'Golden' apresentaram os maiores teores de todos os nutrientes, com exceção do teor de $\mathrm{Zn}$, em que o maior teor deste nutriente foi obtido pelas mudas do 'Tainung 01' (Tabela 4). Canesin e Corrêa (2006), também observaram que as mudas de 'Tainung 02' obtiveram maior teor de $\mathrm{Zn}$ em relação às mudas de 'Sunrise Solo Line 72/12', quando cultivadas em solo.

As mudas do mamoeiro 'Tainung 01' apresentaram, no geral, o menor teor de P na massa seca da parte aérea (Tabela 4). Canesin e Corrêa (2006), ao produzirem mudas de mamoeiros em solo fertilizado com $2,5 \mathrm{~kg} \mathrm{~m}^{-3}$ de superfosfato simples e $0,3 \mathrm{~kg} \mathrm{~m}^{-3}$ de cloreto de potássio, observaram que as mudas de 'Tainung 02 ' apresentaram menor teor de $\mathrm{P}$ em relação às mudas de 'Sunrise Solo Line 72/12'. Yamanishi et al. (2004) também observaram que mudas do 'Tainung 01 ', produzidas em diferentes substratos, apresentaram menor teor de $\mathrm{P}$ em relação às mudas do mamoeiro 'Sunrise Solo'.

Não houve efeito das doses do adubo de liberação lenta sobre os teores de S, B e Zn (Tabela 3), entretanto houve efeito da interação entre os genótipos e as doses do adubo sobre os teores de $\mathrm{S}$ e Zn nas mudas do 'Tainung 01' (Tabelas 5 e 6). Para os outros genótipos, não houve influência das doses do adubo sobre os teores de $\mathrm{Zn}$, fato semelhante ao observado por Oliveira (2000) em mudas do mamoeiro 'Sunrise Solo'. Na garantia do adubo utilizado, não consta a presença de Zn (COMPO DO BRASIL, 2009), e devido ao substrato conter o nutriente, esta quantidade presente pode ter suprido a demanda requerida pelas plantas.

Houve interação significativa entre os genótipos e as doses do adubo para todos os nutrientes avaliados, com exceção do B (Tabela 3). Serrano et al. (2004) também observaram que o incremento nas doses do adubo de liberação lenta, fórmula NPK 1414-14, na produção de mudas cítricas, não interferiu nos teores foliares de B. Apesar de o adubo utilizado neste trabalho conter B na sua formulação, a quantidade desse no substrato parece ter suprido também a demanda desse nutriente requerida pelas mudas de mamoeiro.

O aumento das doses do adubo de liberação lenta, fórmula NPK $(\mathrm{Mg})$ 13-06-16 $(1,4)$, proporcionou aumentos lineares nos teores de $\mathrm{N}$ e $\mathrm{K}$, em todos os genótipos de mamoeiro (Tabela 5). Entretanto, para o teor de $\mathrm{N}$, foi observado que a resposta de maior ajuste foi a equação polinomial quadrática, indicando, porém, doses máximas acima das utilizadas neste experimento para os genótipos 'Golden', 'Calimosa' e 'Tainung 01'.

Ao aumentar as doses do adubo, houve também acréscimo linear nos teores de $\mathrm{Pe} \mathrm{S}$ na massa seca das mudas do 'Tainung 01'; no teor de $\mathrm{Mg}$ nas mudas do 'INCAPER 39'; e no teor de Mn nas mudas do 'Golden', 'Calimosa', 'INCAPER 09' e 'INCAPER 39' (Tabelas 5 e 6). Esses efeitos podem ser atribuídos ao fato de o adubo utilizado conter esses nutrientes em sua formulação e também pela resposta positiva dos respectivos genótipos à aplicação dos mesmos. Ademais, adubos com $\mathrm{N}$-amoniacal tendem a aumentar o teor de Mn nas folhas pelo efeito na disponibilidade (MALAVOLTA, 2006).

Nas mudas do mamoeiro 'Tainung 01', foi observada resposta quadrática para o teor de $\mathrm{Zn}$ na massa seca da parte aérea, indicando que doses do adubo acima de $6,8 \mathrm{~kg} \mathrm{~m}^{-3}$ proporcionaram redução no teor desse nutriente (Tabela 6). Como as mudas desse genótipo apresentaram resposta linear positiva 
entre o aumento das doses do adubo e o teor de $\mathrm{P}$, o decréscimo no teor de $\mathrm{Zn}$ pode estar associado a esse aumento na absorção de $\mathrm{P}$, uma vez que existe uma relação inversa entre esses nutrientes (MALAVOLTA, 2006).

$\mathrm{O}$ aumento nas doses do adubo promoveu decréscimos nos teores de $\mathrm{P}, \mathrm{Fe}$ e $\mathrm{Cu}$ na massa seca da parte aérea das mudas dos mamoeiros 'Golden', 'Calimosa', 'INCAPER 09' e 'INCAPER 39' (Tabelas 5 e 6). Também houve decréscimo nos teores de Ca das mudas de 'Golden', 'Calimosa'e 'INCAPER 39' em relação ao aumento das doses do adubo.

Segundo Malavolta (2006), quando há um aumento acentuado no acúmulo de matéria seca pelas plantas, pode ocorrer uma diminuição aparente nos teores de alguns nutrientes. Neste trabalho, verificamos que, ao aumentar as doses do adubo, houve um aumento no acúmulo de matéria seca das mudas até certo ponto, isto é, uma resposta do tipo quadrática positiva (Tabela 2), enquanto para os teores de $\mathrm{P}, \mathrm{Ca}$, $\mathrm{Mg}, \mathrm{Fe}$ e $\mathrm{Cu}$, a resposta foi do tipo quadrática inversa (Tabelas 5 e 6), mostrando a possível relação entre o acúmulo de matéria seca e a diminuição dos teores desses nutrientes.
Vale ressaltar, também, que o aumento na aplicação de $\mathrm{K}$ e/ou de $\mathrm{N}$-amoniacal tende a diminuir os teores de $\mathrm{Ca}, \mathrm{Mg}$ e $\mathrm{Cu}$ pelas plantas, devido à competição no processo de absorção (MALAVOLTA, 2006).

Os teores médios dos nutrientes na massa seca da parte aérea das mudas dos cinco genótipos de mamoeiro avaliados neste trabalho (Tabela 4) estão dentro ou próximos dos teores-padrão sugeridos por Malavolta et al. (1997) e pelos observados por Almeida et al. (2002), ambos para mamoeiros adultos. Apenas os teores de N, P, Ca e Fe estão um pouco abaixo das referências citadas. Entretanto, se considerarmos os teores máximos observados ou os teores obtidos pelas mudas de todos os genótipos que obtiveram os maiores valores de altura e massa seca total (Tabelas 5 e 6 ), os mesmos atendem aos valores recomendados. Sendo assim, pode-se inferir que, além da diagnose visual, as mudas que apresentaram os maiores valores de crescimento apresentaram, também, adequado estado nutricional.

TABELA 1- Valores de F para as características de crescimento das mudas de cinco genótipos de mamoeiro produzidas em substrato fertilizado com seis doses de um adubo de liberação lenta, fórmula NPK $(\mathrm{Mg})$ 13-06-16 $(1,4)^{(1)}$.

\begin{tabular}{lcccccccc}
\hline $\begin{array}{c}\text { Causas da } \\
\text { Variação }\end{array}$ & G.L. $^{(2)}$ & $\begin{array}{c}\text { ALT } \\
(\mathbf{c m})\end{array}$ & $\begin{array}{c}\text { DC } \\
(\mathbf{m m})\end{array}$ & NF & $\begin{array}{c}\text { CR } \\
(\mathbf{c m})\end{array}$ & $\begin{array}{c}\text { MSPA } \\
(\mathbf{g})\end{array}$ & $\begin{array}{c}\text { MSSR } \\
(\mathbf{g})\end{array}$ & $\begin{array}{c}\text { MST } \\
(\mathbf{g})\end{array}$ \\
\hline Genótipo (G) & $\mathbf{4}$ & $90,6^{*}$ & $23,5^{*}$ & $20,42^{*}$ & $10,5^{*}$ & $14,2^{*}$ & $14,2^{*}$ & $16,4^{*}$ \\
Dose (D) & $\mathbf{5}$ & $892,4^{*}$ & $951,9^{*}$ & $511,1^{*}$ & $95,4^{*}$ & $173,8^{*}$ & $68,5^{*}$ & $162,2^{*}$ \\
G x D & $\mathbf{2 0}$ & $25,7^{*}$ & $5,7^{*}$ & $3,5^{*}$ & $1,6^{\mathrm{ns}}$ & $3,1^{*}$ & $2,0^{*}$ & $2,7^{*}$ \\
Bloco & $\mathbf{2}$ & $67,4^{*}$ & $11,9^{*}$ & $92,9^{*}$ & $0,1^{\mathrm{ns}}$ & $29,6^{*}$ & $19,3^{*}$ & $31,9^{*}$ \\
Resíduo & $\mathbf{1 0 4 8}$ & & & & & & & \\
Total & $\mathbf{1 0 7 9}$ & & & & & & & \\
\hline Média Geral & & $\mathbf{7 , 3}$ & $\mathbf{2 , 0}$ & $\mathbf{5 , 6}$ & $\mathbf{1 4 , 2}$ & $\mathbf{0 , 0 8}$ & $\mathbf{0 , 0 3}$ & $\mathbf{0 , 1 1}$ \\
\hline CV (\%) & & $\mathbf{1 0 , 6}$ & $\mathbf{1 0 , 0}$ & $\mathbf{1 0 , 9}$ & $\mathbf{1 0 , 9}$ & $\mathbf{1 4 , 6}$ & $\mathbf{1 9 , 2}$ & $\mathbf{1 4 , 3}$ \\
\hline
\end{tabular}

(1) * $\mathrm{p} \leq 0,05$ e ${ }^{\mathrm{ns}}$ não significativo;

(2) G.L. = Graus de liberdade; ALT = altura das mudas; DC = diâmetro do caule; NF = número de folhas; CR = comprimento da raiz; $\mathrm{MSPA}=$ massa seca da parte aérea; MSSR = massa seca do sistema radicular, e MST = massa seca total. 
TABELA 2- Efeito das doses de um adubo de liberação lenta, fórmula NPK (Mg) 13-06-16 (1,4), sobre o crescimento de mudas de cinco genótipos de mamoeiro, aos 30 dias após a semeadura.

\begin{tabular}{|c|c|c|c|c|}
\hline Genótipo & Equação de regressão (5\%) & $\begin{array}{l}\text { Coeficiente de } \\
\text { determinação } \\
\mathbf{R}^{2}\end{array}$ & $\begin{array}{c}\text { Dose máxima } \\
\left(\mathrm{kg} \mathrm{m}^{-3}\right)\end{array}$ & $\begin{array}{c}\text { Valor } \\
\text { máximo }\end{array}$ \\
\hline \multicolumn{5}{|c|}{ Altura (cm) } \\
\hline 'Golden’ & $Y=4,602877+0,6942103 x-0,03108730 x^{2}$ & 0,99 & 11,2 & 8,5 \\
\hline 'Calimosa' & $Y=4,234425+1,2677107 x-0,08246826 x^{2}$ & 0,81 & 7,7 & 9,1 \\
\hline 'Incaper 09’ & $Y=4,316468+0,7169603 x-0,03398413 x^{2}$ & 0,99 & 10,5 & 8,1 \\
\hline ‘Tainung 01' & $Y=4,387302+0,9968254 x-0,04279365 x^{2}$ & 0,97 & 11,6 & 10,2 \\
\hline 'Incaper 39’ & $Y=4,274603+0,7786826 x-0,03539683 x^{2}$ & 0,99 & 11,0 & 8,6 \\
\hline \multicolumn{5}{|c|}{ Diâmetro do caule (mm) } \\
\hline 'Golden' & $Y=1,225734+0,2048468 x-0,00892540 x^{2}$ & 0,99 & 11,5 & 2,40 \\
\hline 'Calimosa' & $Y=1,361935+0,2395392 x-0,01330714 x^{2}$ & 0,91 & 9,0 & 2,44 \\
\hline 'Incaper 09' & $Y=1,160555+0,2175111 x-0,01048889 x^{2}$ & 0,98 & 10,4 & 2,29 \\
\hline 'Tainung 01' & $Y=1,105655+0,2494389 x-0,01176032 x^{2}$ & 0,99 & 10,6 & 2,43 \\
\hline 'Incaper 39' & $Y=1,161091+0,2382643 x-0,01207778 x^{2}$ & 0,99 & 9,9 & 2,34 \\
\hline \multicolumn{5}{|c|}{ Número de folhas } \\
\hline 'Golden' & $Y=3,985119+0,4538492 x-0,02007937 x^{2}$ & 0,95 & 11,3 & 6,5 \\
\hline 'Calimosa' & $Y=3,997024+0,5316270 x-0,03087302 x^{2}$ & 0,93 & 8,6 & 6,3 \\
\hline 'Incaper 09' & $Y=3,566468+0,4759921 x-0,02246032 x^{2}$ & 0,98 & 10,6 & 6,1 \\
\hline ‘Tainung 01’' & $Y=3,690476+0,6117460 x-0,03111111 x^{2}$ & 0,96 & 9,8 & 6,7 \\
\hline 'Incaper 39' & $Y=3,825397+0,5687302 x-0,03079365 x^{2}$ & 0,99 & 9,2 & 6,5 \\
\hline \multicolumn{5}{|c|}{ Massa da matéria seca da parte aérea (g) } \\
\hline 'Golden’ & $Y=0,013427+0,0131634 x-0,00052943 x^{2}$ & 0,99 & 12,4 & 0,095 \\
\hline 'Calimosa' & $Y=0,014960+0,0265325 x-0,00163333 x^{2}$ & 0,93 & 8,1 & 0,123 \\
\hline 'Incaper 09' & $Y=0,010664+0,0153365 x-0,00063410 x^{2}$ & 0,99 & 12,1 & 0,103 \\
\hline 'Tainung 01' & $Y=0,007345+0,0209050 x-0,00086762 x^{2}$ & 0,98 & 12,0 & 0,133 \\
\hline 'Incaper 39' & $Y=0,010955+0,0195856 x-0,00096705 x^{2}$ & 0,99 & 10,1 & 0,110 \\
\hline \multicolumn{5}{|c|}{ Massa da matéria seca do sistema radicular (g) } \\
\hline 'Golden' & $Y=0,007446+0,0053986 x-0,00028905 x^{2}$ & 0,97 & 9,3 & 0,033 \\
\hline 'Calimosa' & $Y=0,007446+0,0112944 x-0,00074886 x^{2}$ & 0,88 & 7,5 & 0,050 \\
\hline 'Incaper 09’ & $Y=0,005943+0,0063716 x-0,00038514 x^{2}$ & 0,99 & 8,3 & 0,032 \\
\hline 'Tainung 01' & $Y=0,008702+0,0074944 x-0,00043943 x^{2}$ & 0,91 & 8,5 & 0,041 \\
\hline 'Incaper 39' & $Y=0,008646+0,0061782 x-0,00037400 x^{2}$ & 0,85 & 8,3 & 0,034 \\
\hline \multicolumn{5}{|c|}{ Massa da matéria seca total (g) } \\
\hline 'Golden' & $Y=0,020881+0,0185705 x-0,00081943 x^{2}$ & 0,99 & 11,3 & 0,126 \\
\hline 'Calimosa' & $Y=0,022401+0,0378408 x-0,00238333 x^{2}$ & 0,93 & 7,9 & 0,173 \\
\hline 'Incaper 09’ & $Y=0,016631+0,0216999 x-0,00101886 x^{2}$ & 0,99 & 10,6 & 0,132 \\
\hline ‘Tainung 01’' & $Y=0,016020+0,0284090 x-0,00130790 x^{2}$ & 0,98 & 10,9 & 0,170 \\
\hline 'Incaper 39' & $Y=0,019581+0,0257785 x-0,00134210 x^{2}$ & 0,99 & 9,6 & 0,143 \\
\hline
\end{tabular}


TABELA 3- Valores de F para os teores de macronutrientes $\left(\right.$ dag $\left.\mathrm{kg}^{-1}\right)$ e micronutrientes $\left(\mathrm{mg} \mathrm{kg}^{-1}\right)$ da matéria seca da parte aérea das mudas de cinco genótipos de mamoeiro produzidas em substrato fertilizado com seis doses de um adubo de liberação lenta, fórmula NPK $(\mathrm{Mg}) 13-06-16(1,4)^{(1)}$.

\begin{tabular}{lcccccccccccc}
\hline $\begin{array}{l}\text { Causas } \\
\text { da Variação }\end{array}$ & G.L. & $\mathbf{N}$ & $\mathbf{P}$ & $\mathbf{K}$ & $\mathbf{C a}$ & $\mathbf{M g}$ & $\mathbf{S}$ & $\mathbf{B}$ & $\mathbf{C u}$ & $\mathbf{F e}$ & $\mathbf{M n}$ & $\mathbf{Z n}$ \\
\hline Genótipo (G) & $\mathbf{4}$ & $18,8^{*}$ & $52,3^{*}$ & $15,8^{*}$ & $14,3^{*}$ & $6,7^{*}$ & $8,8^{*}$ & $3,9^{*}$ & $64,3^{*}$ & $14,7^{*}$ & $40,9^{*}$ & $13,5^{*}$ \\
Dose (D) & $\mathbf{5}$ & $310,0^{*}$ & $31,1^{*}$ & $102,6^{*}$ & $4,8^{*}$ & $27,9^{*}$ & $2,3^{\mathrm{ns}}$ & $2,1^{\mathrm{ns}}$ & $47,5^{*}$ & $19,5^{*}$ & $263,2^{*}$ & $0,2^{\mathrm{ns}}$ \\
G x D & $\mathbf{2 0}$ & $2,9^{*}$ & $4,7^{*}$ & $2,9^{*}$ & $5,2^{*}$ & $2,3^{*}$ & $3,0^{*}$ & $1,4^{\mathrm{ns}}$ & $5,6^{*}$ & $2,2^{*}$ & $8,8^{*}$ & $1,9^{*}$ \\
Bloco & $\mathbf{2}$ & $11,3^{*}$ & $21,4^{*}$ & $2,9^{*}$ & $3,6^{*}$ & $4,9^{*}$ & $6,3^{*}$ & $20,8^{*}$ & $10,1^{*}$ & $0,6^{\mathrm{ns}}$ & $6,6^{*}$ & $0,1^{\mathrm{ns}}$ \\
Resíduo & $\mathbf{5 8}$ & & & & & & & & & & & \\
Total & $\mathbf{8 9}$ & & & & & & & & & & & \\
\hline Média Geral & & $\mathbf{3 , 8}$ & $\mathbf{0 , 3}$ & $\mathbf{4 , 1}$ & $\mathbf{1 , 4}$ & $\mathbf{1 , 1}$ & $\mathbf{0 , 5}$ & $\mathbf{1 0 3 , 9}$ & $\mathbf{1 2 , 4}$ & $\mathbf{1 7 3 , 6}$ & $\mathbf{6 5 , 5}$ & $\mathbf{4 3 , 9}$ \\
\hline C.V. (\%) & & $\mathbf{6 , 1}$ & $\mathbf{6 , 5}$ & $\mathbf{7 , 5}$ & $\mathbf{5 , 4}$ & $\mathbf{5 , 6}$ & $\mathbf{8 , 1}$ & $\mathbf{2 1 , 9}$ & $\mathbf{6 , 7}$ & $\mathbf{1 7 , 4}$ & $\mathbf{6 , 1}$ & $\mathbf{3 7 , 6}$ \\
\hline
\end{tabular}

(1) * $\mathrm{p} \leq 0,05 \mathrm{e}^{\mathrm{ns}}$ não significativo.

TABELA 4- Teores médios de nutrientes na matéria seca da parte aérea das mudas de cinco genótipos de mamoeiro, produzidas em substrato fertilizado com um adubo de liberação lenta, fórmula NPK $(\mathrm{Mg})$ 13-06-16 $(1,4)^{(1)}$.

\begin{tabular}{|c|c|c|c|c|c|c|c|c|c|c|c|}
\hline Gen & $\mathbf{N}$ & $\mathbf{P}$ & $\mathbf{K}$ & $\mathrm{Ca}$ & Mg & $\mathbf{S}$ & B & u & $\mathbf{F e}$ & Mn & $\mathrm{Zn}$ \\
\hline & \multicolumn{11}{|c|}{$\ldots$. } \\
\hline & & & & & & & & & & & \\
\hline ' $\mathrm{Ca}$ & , & & & & & & & & & & \\
\hline 'In & 3,7 & & & & & & & & & & \\
\hline Tai & $3,6 \mathrm{~b}$ & 0 & & 1 & & 0 & & & $146 \mathrm{~b}$ & & 67 \\
\hline 'Incaper 39' & $3,8 \mathrm{~b}$ & $0,25 \mathrm{~b}$ & & & & & & & $145 \mathrm{~b}$ & $61,1 \mathrm{c}$ & $47,1 \mathrm{~b}$ \\
\hline
\end{tabular}

(1) Médias seguidas pela mesma letra nas colunas não diferem estatisticamente, pelo teste de Tukey, a 5\% de probabilidade. 
TABELA 5- Efeito das doses de um adubo de liberação lenta, fórmula NPK $(\mathrm{Mg})$ 13-06-16 $(1,4)$, sobre o teor de macronutrientes na massa seca da parte aérea de mudas de mamoeiro, aos 30 dias após a semeadura.

\begin{tabular}{|c|c|c|c|c|}
\hline Genótipo & Equação de regressão (5\%) & $\begin{array}{l}\text { Coeficiente de } \\
\text { determinação } \\
\mathbf{R}^{2}\end{array}$ & $\begin{array}{c}\text { Dose máxima } \\
\left(\mathrm{kg} \mathrm{m}^{-3}\right)\end{array}$ & $\begin{array}{l}\text { Valor máximo } \\
\left(\text { dag } \mathrm{kg}^{-1}\right)\end{array}$ \\
\hline \multicolumn{5}{|c|}{ 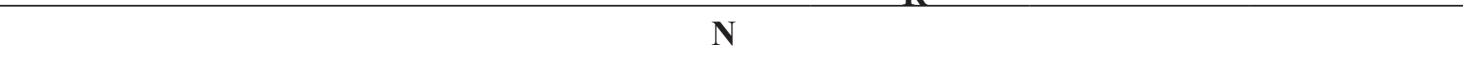 } \\
\hline 'Golden' & $Y=2,331786+0,4368905 x-0,01480952 x^{2}$ & 0,95 & 14,8 & 5,71 \\
\hline 'Calimosa' & $Y=1,945833+0,3734333 x-0,00926667 x^{2}$ & 0,98 & 20,1 & 5,71 \\
\hline 'Incaper 09’ & $Y=2,170000+0,3910667 x-0,01680000 x^{2}$ & 0,94 & 11,6 & 4,45 \\
\hline 'Tainung 01' & $Y=2,295000+0,2787333 x-0,00706667 x^{2}$ & 0,99 & 19,7 & 5,04 \\
\hline 'Incaper 39’ & $Y=2,015833+0,5126333 x-0,02526667 x^{2}$ & 0,96 & 10,1 & 4,57 \\
\hline \multicolumn{5}{|c|}{ 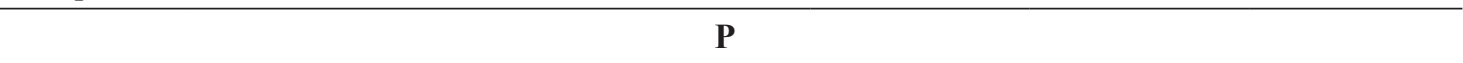 } \\
\hline 'Golden' & $Y=0,378940-0,2452619 x+0,01532381 x^{2}$ & 0,91 & 0,0 & 0,38 \\
\hline 'Calimosa' & $Y=0,293179-0,1748238 x+0,01410476 x^{2}$ & 0,87 & 0,0 & 0,29 \\
\hline 'Incaper 09’ & $Y=0,309440-0,2288905 x+0,01595238 x^{2}$ & 0,92 & 0,0 & 0,31 \\
\hline 'Tainung 01' & $Y=0,2159365+0,0311238 x$ & 0,88 & 12,5 & 0,26 \\
\hline 'Incaper 39' & $Y=0,310619-0,2284571 x+0,01493333 x^{2}$ & 0,68 & 0,0 & 0,31 \\
\hline \multicolumn{5}{|c|}{$\mathbf{K}$} \\
\hline 'Golden' & $Y=2,863492+0,2129524 x$ & 0,98 & 12,5 & 5,52 \\
\hline 'Calimosa' & $Y=2,673810+0,1601905 x$ & 0,91 & 12,5 & 4,68 \\
\hline 'Incaper 09’ & $Y=3,070635+0,1304762 x$ & 0,86 & 12,5 & 4,70 \\
\hline 'Tainung 01' & $Y=3,522222+0,1426667 x$ & 0,67 & 12,5 & 5,31 \\
\hline 'Incaper 39' & $Y=2,945238+0,1900952 x$ & 0,95 & 12,5 & 5,32 \\
\hline \multicolumn{5}{|c|}{$\mathrm{Ca}$} \\
\hline 'Golden' & $Y=1,571905-0,0157714 x$ & 0,61 & 0,0 & 1,57 \\
\hline 'Calimosa' & $Y=1,440476-0,0404000+0,00285714 x^{2}$ & 0,74 & 0,0 & 1,44 \\
\hline 'Incaper 09' & $Y=1,384643-0,0474143+0,0058381 x^{2}$ & 0,72 & 12,5 & 1,70 \\
\hline 'Incaper 39' & $\mathrm{Y}=1,542857-0,0092571 \mathrm{x}$ & 0,47 & 0,0 & 1,54 \\
\hline \multicolumn{5}{|c|}{ Mg } \\
\hline 'Golden' & $Y=1,138690-0,0267381 x+0,00311429 x^{2}$ & 0,85 & 12,5 & 1,29 \\
\hline 'Calimosa' & $Y=1,139405-0,0569952 x+0,00465714 x^{2}$ & 0,88 & 12,5 & 1,15 \\
\hline 'Incaper 09’ & $Y=1,055833-0,0464524 x+0,00528571 x^{2}$ & 0,74 & 12,5 & 1,30 \\
\hline 'Tainung 01' & $Y=1,089643-0,0269095 x+0,00300000 x^{2}$ & 0,91 & 12,5 & 1,22 \\
\hline 'Incaper 39' & $Y=1,019683+0,0122286 x$ & 0,68 & 12,5 & 1,17 \\
\hline \multicolumn{5}{|c|}{$\mathbf{S}$} \\
\hline 'Tainung 01' & $Y=0,436667+0,0101333 x$ & 0,72 & 12,5 & 0,56 \\
\hline
\end{tabular}


TABELA 6- Efeito das doses de um adubo de liberação lenta, fórmula NPK $(\mathrm{Mg})$ 13-06-16 $(1,4)$, sobre o teor de micronutrientes na matéria seca da parte aérea de mudas de mamoeiro, aos 30 dias após a semeadura.

\begin{tabular}{|c|c|c|c|c|}
\hline Genótipo & Equação de regressão (5\%) & $\begin{array}{l}\text { Coeficiente de } \\
\text { determinação } \\
\mathbf{r}^{2}\end{array}$ & $\begin{array}{c}\text { Dose máxima } \\
\left(\mathrm{kg} \mathrm{m}^{-3}\right)\end{array}$ & $\begin{array}{c}\text { Valor máximo } \\
\left(\mathrm{mg} \mathrm{kg}^{-1}\right)\end{array}$ \\
\hline \multicolumn{5}{|c|}{ Zn } \\
\hline 'Tainung 01 ' & $Y=35,648571+15,7634672 x-1,16350479 x^{2}$ & 0,75 & 6,8 & 89,04 \\
\hline \multicolumn{5}{|c|}{$\mathrm{Fe}$} \\
\hline 'Golden' & $Y=259,047497-28,4030515 x+1,84435234 x^{2}$ & 0,73 & 0,0 & 259,05 \\
\hline 'Calimosa' & $Y=269,454167-29,3841376 x+2,12637134 x^{2}$ & 0,84 & 0,0 & 269,45 \\
\hline 'Incaper 09' & $Y=287,677025-42,0529380 x+2,73978092 x^{2}$ & 0,99 & 0,0 & 287,68 \\
\hline 'Incaper 39' & $Y=197,627502-18,2940636 x+1,08442872 x^{2}$ & 0,85 & 0,0 & 197,63 \\
\hline \multicolumn{5}{|c|}{ Mn } \\
\hline 'Golden' & $Y=49,986032+3,9756573 x$ & 0,76 & 12,5 & 99,68 \\
\hline 'Calimosa' & $Y=39,684603+4,1918856 x$ & 0,77 & 12,5 & 92,08 \\
\hline 'Incaper 09’ & $Y=47,452540+1,8981713$ & 0,42 & 12,5 & 71,18 \\
\hline 'Incaper 39' & $Y=44,203492+2,7001523$ & 0,80 & 12,5 & 77,96 \\
\hline \multicolumn{5}{|c|}{$\mathbf{C u}$} \\
\hline 'Golden' & $Y=18,437857-1,3425618 x+0,08603809 x^{2}$ & 0,84 & 0,0 & 18,44 \\
\hline 'Calimosa' & $Y=15,308929-1,0641571 x+0,07162857 x^{2}$ & 0,92 & 0,0 & 15,31 \\
\hline 'Incaper 09' & $Y=14,926905-1,4077047 x+0,10386667 x^{2}$ & 0,92 & 0,0 & 14,93 \\
\hline 'Incaper 39' & $Y=15,280357-1,4649192 x+0,08922858 x^{2}$ & 0,93 & 0,0 & 15,28 \\
\hline
\end{tabular}

\section{CONCLUSÕES}

1-Há diferença de crescimento e dos teores de nutrientes entre os genótipos de mamoeiros 'Golden', 'Calimosa', 'Tainung 01', 'INCAPER 09' e 'INCAPER 39'.

2-As doses do adubo de liberação lenta, fórmula NPK $(\mathrm{Mg})$ 13-06-16 $(1,4)$, que proporciona a maior altura das mudas de mamoeiro, são $11,2 \mathrm{~kg}$ $\mathrm{m}^{-3}$ para o 'Golden'; 7,7 $\mathrm{kg} \mathrm{m}^{-3}$ para o 'Calimosa'; $10,5 \mathrm{~kg} \mathrm{~m}^{-3}$ para o 'INCAPER 09'; 11,6 $\mathrm{kg} \mathrm{m}^{-3}$ para o 'Tainung 01', e 11,0 $\mathrm{kg} \mathrm{m}^{-3}$ para o 'INCAPER 39'.

3-As doses do adubo de liberação lenta, fórmula NPK $(\mathrm{Mg})$ 13-06-16 $(1,4)$, que proporciona a maior massa seca total das mudas de mamoeiro, são $11,3 \mathrm{~kg} \mathrm{~m}^{-3}$ para o 'Golden'; 7,9 $\mathrm{kg} \mathrm{m}^{-3}$ para o 'Calimosa'; 10,6 $\mathrm{kg} \mathrm{m}^{-3}$ para o 'INCAPER 09'; $10,9 \mathrm{~kg} \mathrm{~m}^{-3}$ para o 'Tainung 01 ', e 9,6 $\mathrm{kg} \mathrm{m}^{-3}$ para o 'INCAPER 39'.

4-Nas doses do adubo de liberação lenta, citadas acima, as mudas de mamoeiro apresentam adequado estado nutricional.

\section{AGRADECIMENTO}

Ao Técnico Agrícola Elieuder Celim da Caliman Agrícola $\mathrm{S} / \mathrm{A}$, pelo apoio à realização do experimento.

\section{REFERÊNCIAS}

ALMEIDA, F.T.; BERNARDO, S.; MARINHO, C.S.; MARIN, S.L.D.; SOUSA, E.F. Teores de nutrientes do mamoeiro 'Improved Sunrise Solo 72/12' sob diferentes lâminas de irrigação, no Norte Fluminense. Revista Brasileira de Fruticultura, Jaboticabal, v.24, n.2, p.547-551, 2002.

CANESIN, R.C.F.S.; CORREAA, L.S. Uso de esterco associado à adubação mineral na produção de mudas de mamoeiro (Carica papaya L.). Revista Brasileira de Fruticultura, Jaboticabal, v.28, n.3, p.481-486, 2006.

COMPO DO BRASIL. Basacote Plus. Disponível em: $<$ http://www.international.compo.com $>$. Acesso em: 27 abr. 2009. 
DANTAS, J.L.L.; DANTAS, A.C.V.L.; LIMA, J.F. Mamoeiro. In: BRUCKNER, C.H. (Ed.) Melhoramento de fruteiras tropicais. Viçosa: Editora UFV, 2002. p.309-349.

FRAIFE FILHO, G.A.; DANTAS, J.L.L.; LEITE, J.B.V.; OLIVEIRA, J.R.P. Avaliação de variedades de mamoeiro no extremo sul da Bahia. Magistra, Cruz das Almas, v.13, n.1, p.37-41, 2001.

IBGE - Instituto Brasileiro de Geografia e Estatística. Produção Agrícola Municipal. Disponível em: $<$ http//www.sidra.ibge.gov.br $>$. Acesso em: $01 \mathrm{dez}$. 2008.

KÄMPF, A. Evolução e perspectivas do crescimento do uso de substratos no Brasil. In: BARBOSA, J.G.; MARTINEZ, H.E.P.; PEDROSA, M.W.; SEDIYAMA, M.A.N. (Ed.). Nutrição e adubação de plantas cultivadas em substrato. Viçosa: UFV, 2004. p.3-10.

LANA, R.M.Q.; SANTOS, C.M.; SANTOS, V.L.M.; BARBIZAN, E.L.; MENDES, A.F. Utilização de diferentes substratos e de fertilizantes de liberação lenta na produção de mudas do cafeeiro em saquinhos. Revista Ceres, Viçosa, MG, v.49, n.286, p.577-586, 2002.

MALAVOLTA, E. Manual de nutrição mineral de plantas. São Paulo: Agronômica Ceres, 2006. 638p.

MALAVOLTA, E.; VITTI, G.C.; OLIVEIRA, S.A. Avaliação do estado nutricional das plantas: princípios e aplicações. 2. ed. Piracicaba: POTAFÓS, 1997. 319p.

MARANA, J.P.; MIGLIORANZA, E.; FONSECA, E.P.; KAINUMA, R.H. Índices de qualidade e crescimento de mudas de café produzidas em tubetes. Ciência Rural, Santa Maria, v.38, n.1, p.39-45, 2008.

MARINHO, C.S.; MONNERAT, P.H.; CARVALHO, A.J.C.; MARINS, S.L.D.; VIEIRA, A. Análise química do pecíolo e limbo foliar como indicadora do estado nutricional dos mamoeiros 'Solo' e 'Formosa'. Scientia Agricola, Piracicaba, v.59, n.2, p.373-381, 2002.

MENDONÇA, V.; ARAÚJO NETO, S.E.; RAMOS, J.D.; PIO, R.; GONTIJO, T.C.A. Diferentes substratos e recipientes na formação de mudas de mamoeiro 'Sunrise Solo'. Revista Brasileira de Fruticultura, Jaboticabal, v.25, n.1, p.127-130, 2003.
MENDONÇA, V.; RAMOS, J.D.; DANTAS, D.J.; MARTINS, P.C.C.; GONTIJO, T.C.A.; PIO, R. Efeitos de doses de osmocote e dois tipos de substratos no crescimento de mudas de mamoeiro 'Formosa'. Revista Ceres, Viçosa, MG, v.51, n.296, p.467476, 2004.

OLIVEIRA, P.R.A. Efeitos do fósforo e zinco na nutrição e crescimento de mudas de mamoeiro e mangabeira. 2000. 184f. Tese (Doutorado) - Universidade Federal de Lavras, Lavras, 2000.

PEREIRA, W.E.; LIMA, S.F.; PAULA, L.B.; ALVAREZ, V.; V.H. Crescimento e composição mineral de mudas de maracujazeiro em função de doses de osmocote em dois tipos de substratos. Revista Ceres, Viçosa, v.47, n.271, p.311-324, 2000.

SERRANO, L.A.L.; MARINHO, C.S.; CARVALHO, A.J.C.; MONNERAT, P.H. Efeito de sistemas de produção e doses de adubo de liberação lenta no estado nutricional de porta-enxerto cítrico. Revista Brasileira de Fruticultura, Jaboticabal, v.26, n.3, p.524-528, 2004.

SERRANO, L.A.L.; SILVA, C.M.M.; OGLIARI, J.; CARVALHO, A.J.C.; MARINHO, C.S.; DETMANN, E. Utilização de substrato composto por resíduos da agroindústria canavieira para produção de mudas de maracujazeiro-amarelo. Revista Brasileira de Fruticultura, Jaboticabal, v.28, n.3, p.487-491, 2006.

SILVA, F.C. Manual de análises químicas de solos, plantas e fertilizantes. Brasília: EMBRAPA Comunicação para transferência de tecnologia, 1999.370p.

TOMASZEWSKA, M.; JAROSIEWICZ, A.; KARAKULSKI, K. Physical and chemical characteristics of polymer coatings in CRF formulation. Desalination, Hopkinton, v.146, p.319-323, 2002.

VENTURA, J.A.; COSTA, H.; TATAGIBA, J.S. Doenças do mamoeiro. In: MANICA, I.; MARTINS, D.S.; VENTURA, J.A. (Ed.) Mamão: tecnologia de produção, pós-colheita, exportação, mercados. Porto Alegre: Cinco Continentes, 2006. cap.9. p.207-241.

YAMANISHI, O.K.; FAGUNDES, G.R.; MACHADO FILHO, J.A.; VALONE, G.V. Efeito de diferentes substratos e duas formas de adubação na produção de mudas de mamoeiro. Revista Brasileira de Fruticultura, Jaboticabal, v.26, n.2, p.276-279, 2004. 